\section{LA-UR-O $3-2939$}

Approved for public release;

distribution is unlimited.

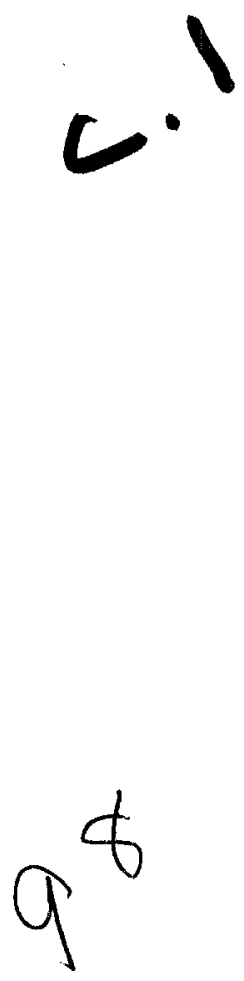

Title:

NETWORK FAULT TOLERANCE IN LA-MPI

Author(s):

Rob T Aulwes, David J. Daniel, Nehal N. Desai,

Richard L.Graham, L. Dean Risinger, Mitchel W. Sukalski and Mark A. Taylor

Submitted to:

\section{European MPI/PVM User's Meeting,}

Venice, Italy

Sept 2003

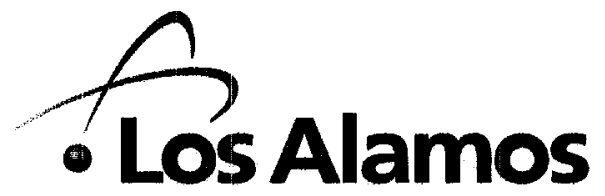

NATIONAL LABORATORY

Los Alamos National Laboratory, an affirmative action/equal opportunity employer, is operaterny the University of California for the U.S. Department of Energy under contract W-7405-ENG-36. By acceptance of this article, the publisher recognizes that the U.S. Government retains a nonexclusive, royalty-free license to publish or reproduce the published form of this contribution, or to allow others to do so, for U.S. Government purposes. Los Alamos National Laboratory requests that the publisher identify this article as work performed under the auspices of the U.S. Department of Energy. Los Alamos National Laboratory strongly supports academic freedom and a researcher's right to publish; as an institution, however, the Laboratory does not endorse the viewpoint of a publication or guarantee its technical correctness. 


\title{
Network Fault Tolerance in LA-MPI
}

\author{
Rob T. Aulwes, David J. Daniel, Nehal N. Desai, \\ Richard L. Graham, L. Dean Risinger,

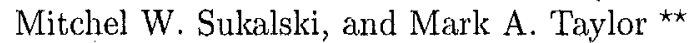 \\ Los Alamos National Laboratory, Advanced Computing Laboratory, \\ MS-B287, P. O. Box 1663, Los Alamos NM 87545, USA \\ lampi-support@lanl.gov \\ http://ww, acl.lanl.gov/la-mpi/
}

\begin{abstract}
LA-MPI is a high-performance, network-fault-tolerant implementation of MPI designed for terascale clusters that are inherently unreliable due to their very large number of system components and to trade-offs between cost and performance. This paper reviews the architectural design of LA-MPI, focusing on our approach to guaranteeing data integrity. We discuss our network data path abstraction that makes LA-MPI highly portable, gives high-performance through message striping, and most importantly provides the basis for network fault tolerance. Finally we include some performance numbers for the Quadrics and UDP network paths.
\end{abstract}

\section{Introduction}

LA-MPI is an implementation of the Message Passing Interface (MPI) $[1,2]$ motivated by a growing need for fault tolerance at the software level in large high-performance computing (HPC) systems.

This need is caused by the sheer number of components present in modern HPC systems, particularly clusters. The individual compontents - processors, memory modules, network interface cards (NICs), etc. - are typically manufactured to tolerances adequate for small or desktop systems. When aggregated into a large HPC system, however, system-wide error rates may be too great to successfully complete a long application run [3]. For example, a network device may have an error rate which is perfectly acceptable for a desktop system, but not in a cluster of thousands of nodes, which must run error free for many hours or even days to complete a scientific calculation.

LA-MPI has two primary goals: network fault tolerance and high performance. Fortunately these goals are partially complimentary, since the flexible approach we take to the use of redundant network data paths to support fault

** Los Alamos report LA-UR-03-XXXX. Los Alamos National Laboratory is operated by the University of California for the National Nuclear Security Administration of the United States Department of Energy under contract W-7405-ENG-36. Project support was provided through ASCI/PSE and the Los Alamos Computer Science Institute. 


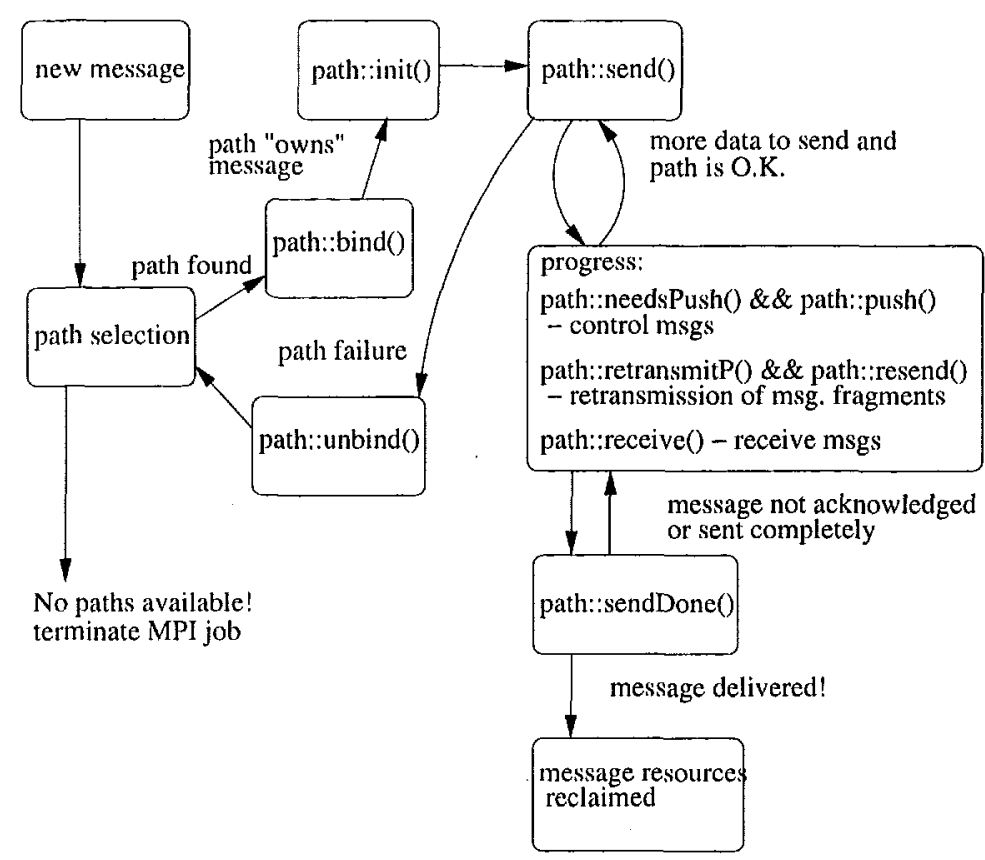

Fig. 1. Message-Path Interactions.

tolerance also allows LA-MPI to exploit all the available network bandwidth in a network-device-rich system by data striping (of both messages and message fragments).

Other important features of LA-MPI include an open source license, standards compliance (MPI version 1.2 integrated with ROMIO [4] for MPI-IO v2 support), thread safety, and portability to many operating systems, processor architectures and network devices.

In the following sections we will review the architecture of LA-MPI emphasizing the important role of our network data path abstraction. A detailed discussion of LA-MPI's data integrity protocol is given in section 3 , while a selection of performance results are presented in section 4 .

\section{Architecture}

At a high level, LA-MPI's architecture falls into two layers: an upper MPI layer, implementing the full richness of the MPI standard, and a lower User Level Messaging (ULM) layer that provides a simpler reliable message passing API. Looking deeper, ULM is itself composed of two layers: a Memory and Message Layer (MML), and a Send and Receive Layer (SRL). The MML consists of code common to all systems and data paths, while the SRL is highly device-specific. 
Before discussing these layers and their interaction in more detail, it is helpful to discuss what types of network fault tolerance are provided by LA-MPI. We distinguish two separate functionalities: (a) guaranteed data integrity of delivered messages; and (b) the ability to fail-over from one network device to another if the first is generating too many errors. Both of these require that we are able to treat each available network device on an equal footing, and this has led us to developing an abstraction called a network data path object or, more succinctly, a path.

A path is an abstraction of lower-level network transports and devices that, are available to LA-MPI. Each path can represent a single network adapter, or a set of common adapters, or even a cornmon protocol over many different network adapters. Currently, paths are implemented for the user datagram protocol [5] (UDP over all IP-enabled devices), Quadrics Elan3 [6] remote direct memory access (RDMA), and Myrinet GM [7], and is currently being developed for Infiniband [8] (Mellanox HCA Verbs [9]). In all of our current paths except UDP/IP, which treats multiple network adapters as a single Internet Protocol "device", multiple network adapters are used by a single path instantiation, if they exist on the machine.

The path object provides the interface between the portable common code of the MML and device-specific SRL. The interaction of the MML with the various paths is described schematically in figure 1.

In the next section we give an in-depth discussion of one aspect of network fault tolerance in LA-MPI, namely support for reliable message delivery, that is, the guaranteed integrity of delivered datia. The other aspects of LA-MPI's architecture are described in more detail elsewhere [10].

\section{Reliability}

Unlike many MPI libraries that consider all underlying communication perfectly reliable, LA-MPI optionally supports sender-side retransmission of messages by checking the unacknowledged list every 5 seconds (adjustable at compile time) for message send descriptors that have exceeded their timeout periods. This retransmission scheme is appropriate for low error rate environments, typical of most clusters. Each network transport is responsible for arranging to retransmit the necessary fragments. Each fragment's retransmission time is calculated using a truncated exponential back-off scheme; this avoids resource exhaustion at a receiving process that is busy doing non-MPI computation. Fragments that must be retransmitted are moved from the Frags ToAck list to the Frags ToSend list, and the associated message send descriptor is placed on the incomplete list.

Each network transport is also responsible for providing a main memory-tomain memory 32-bit additive checksum or 32-bit cyclic redundancy code (CRC), if it is needed. This checksum/CRC protects against network and I/O bus corruption, and is generated at the same time data is copied, if at all possible. By delaying checksumming to avoid wasting memory bandwidth, a received fragment is not necessarily a deliverable, or uncorrupted, fragment. 


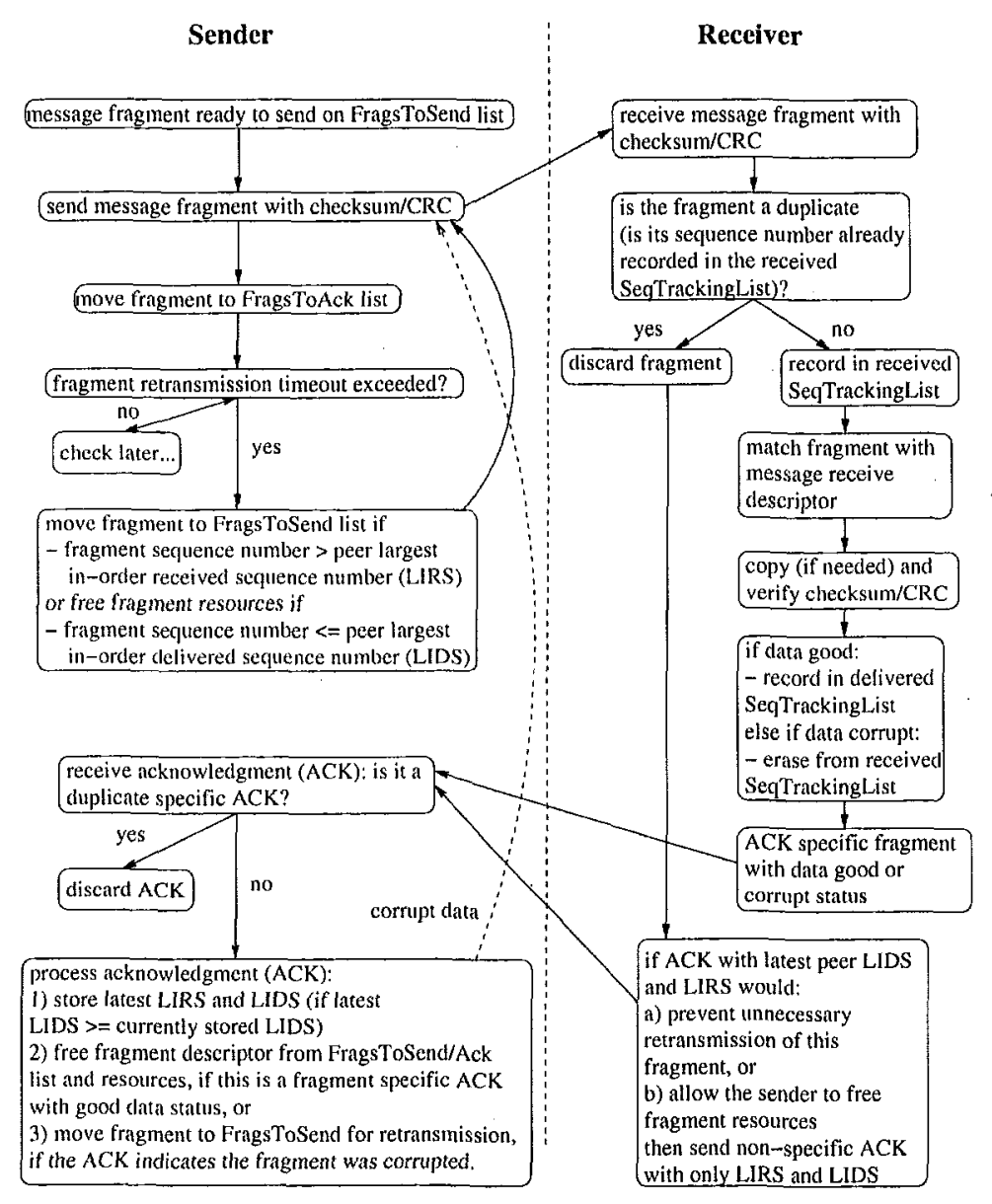

Fig. 2. Retransmission and Checksumming.

Several MML generic features aid in the implementation of this retransmission and checksumming scheme. Every fragment is assigned a monotonically increasing 64-bit sequence number between a given (sender, receiver) pair of processes. These sequence numbers are recorded by the receiving process in a special object, SeqTrackingList, as an ordered set of non-contiguous ranges of sequence numbers; these lists use internal hint pointers to exploit any temporal locality in accessing these lists to minimize access overhead. The receiver maintains two SeqTrackingList lists for each peer with which it communicates to distinguish between fragments that have been received, and those that have been received and delivered successfully (i.e., no data corruption). Duplicate fragments are easily detected by checking the received fragment's sequence number against the received SeqTrackingList. 
Upon processing fragment acknowledgments from a receiver, a sender will store two special values that are carried in every acknowledgment: the largest in-order peer received sequence number (LIRS), and the largest in-order peer delivered sequence number (LIDS). The LIRS is used to prevent the retransmission of fragments that have been received, but whose data integrity has not been checked yet; it may increase or decrease over time, depending upon transmission and I/O bus errors. The LIDS is used to free any fragments whose acknowledgment was lost. The LIDS is always less than or equal to the LIR.S. Figure 2 shows the interaction of these sequence numbers, the retransmission scheme, and checksumming.

\section{Performance}

\section{Conclusions}

With the rise of terascale distributed computing environments consisting of thousands of processors and network adapters, the need for fault tolerant software has become critical to their successful use. Negligible component error and failure rates in small to medium size clusters are no longer negligible in these large clusters, due to their complexity, sheer number of components, and amount of data transferred. LA-MPI addresses the network-related challenges of this environment by providing a production-quality, reliable, high-performance Message Passing Interface (MPI) library for applications capable of (a) surviving network and I/O bus data corruption and loss, and (b) surviving network hardware and software failure if other connectivity is available. In this paper, we have presented an overview of LA-MPI's design and implementation.

LA-MPI is currently available as open source software under an LGPL license. It currently runs on Linux (i686 and Alpha processors), HP's Tru64 (Alpha only), SGI's IRIX 6.5 (MIPS), and Apple's Mac OS X (PowerPC). It supports shared memory, UDP/IP, Quadrics Elan3 RDMA, HIPPI-800 OS bypass (IRIX only), and current work is progressing on Myrinet GM and Infiniband (Mellanox HCA Verbs) communications support. LA-MPI supports job spawning and control with Platform LSF, Quadrics RMS (Tru64 only), Bproc [11], and standard BSD rsh. Please send email to lampi-support@lanl.gov for more information [12]. All fault tolerance features described in this paper have been fully implemented, except for on-going work on automatic network fail-over support.

Future papers will present full performance studies of design trade-offs as part of our on-going optimization of the library. Future development efforts will address:

- the implementation of a fault-tolerant, scalable, administrative network for job control, standard I/O redirection, and MPI wire-up;

- the implementation of process fault-tolerance in the face of multiple process failures; 
the implementation of dynamic topology reconfiguration and addition of MPI processes to support dynamic process migration and MPI-2 dynamic processes.

\section{References}

1. Message Passing Interface Forum. MPI: A Message Passing Interface Standard. Technical report, 1994.

2. Message Passing Interface Forum. MPI-2.0: Extensions to the Message-Passing Interface. Technical report, 1997.

3. C. Partridge, J. Hughes, and J. Stone. Performance of checksums and CRCs over real data. Computer Communication Review, v. 25 n. 4:68-76, 1995.

4. Rajeev Thakur, William Gropp, and Ewing Lusk. Users Guide for ROMIO: A High-Performance, Portable MPI-IO Implementation. Mathematics and Computer Science Division, Argonne National Laboratory, October 1997. ANL/MCS-TM234.

5. J. Postel. RFC 768: User Datagram Protocol, August 1980.

6. Fabrizio Petrini, Wu-Chun Feng, Adolfy Hoisie, Salvador Coll, and Eitan Frachtenberg. The Quadrics network: High-performance clustering technology. IEEE Micro, v. 22 n. 1:46-57, 2002.

7. Myricom, Inc. http://www.myri.com/.

8. Infiniband Trade Association. http://www.infinibandta.org/home.

9. Mellanox Technologies, Inc. http://www.mellänox.com/,

10. Rob T. Aulwes, David J. Daniel, Nehal N. Desai, Richard L. Graham, L. Dean Risinger, and Mitchel W. Sukalski. LA-MPI: The design and implementation of a network-fault-tolerant MPI for terascale clusters. Technical Report LA-UR-030939, Los Alamos National Laboratory, 2003.

11. Advanced Computing Laboratory, Los Alamos National Laboratory. http://public.lanl.gov/cluster/index.html.

12. Advanced Computing Laboratory, Los Alamos National Laboratory. http://wwu. acl.lanl.gov/la-mpi. 